differences in disease development. Female CD103DC have decreased ability to convert conventional CD4 cells to Foxp $3^{+}$ Tregs due to defects in retinoic acid (RA) production; female macrophages have decreased ability to mediate efferocytosis and defects in genes associated with PPAR $\square$ and LXR signaling. Male cecal transplants can restore function of both CD103DC and macrophages in female recipients. Metabolomics analysis of feces revealed that males have higher phytanic acid (PA), an RXR/PPAR $\square / L X R$ agonist. Treatment of female mice in vivo with $\mathrm{PA}$ delays disease to some extent and restores both CD103DC and macrophage function. PA also restores CD103DC and macrophage function when added to female cells directly in vitro. PA enhances CD103DC function by restoring RA production via the RXR pathway and macrophage efferocytosis via the PPAR $\square$ and LXR pathways in female mice.

Conclusions We have identified specific gut microbiota taxa and metabolite candidates from lupus-resistant male mice that have immunomodulatory activity and the potential to mitigate disease in lupus-prone female mice. Disease mitigation may be mediated through an increase in CD103DC function and macrophages efferocytosis.

Acknowledgments Funded by ALR and NIH-R01AR067188. 16S rRNA gene sequencing was performed by GTAC, Washington University, St. Louis. Mass spectrometry was performed by CREAM, University of Louisville, Louisville.

\section{ACELLULAR ARTIFICIAL ANTIGEN-PRESENTING CELLS INDUCE DISEASE-PROTECTIVE IMMUNOREGULATORY CELLS IN LUPUS}

${ }^{1}$ Antonio La Cava*, 'David A Horwitz. 'University of California Los Angeles, Los Angeles, CA, USA; ${ }^{2}$ General Nanotherapeutics, Santa Monica, CA, USA

\subsection{6/lupus-2021-lupus21century.83}

Background T regulatory cells (Tregs) provide beneficial effects in the suppression of autoimmune responses in systemic lupus erythematosus (SLE). We recently showed that poly(lactic-coglycolic acid) PLGA nanoparticles (NPs) loaded with IL-2 and TGF- $\beta$ could induce $\mathrm{CD}^{+}{ }^{+}$and $\mathrm{CD} 8^{+}$Foxp $3^{+}$Tregs in vitro and in vivo, with resulting protective effects in mice that develop lupus-like disease following the transfer of donor $\mathrm{T}$ cells from $\mathrm{DBA} / 2$ mice into $(\mathrm{C} 57 \mathrm{BL} / 6 \times \mathrm{DBA} / 2) \mathrm{F}_{1}$ (BDF1) recipients.

Methods We produced NPs encapsulating IL-2 as acellular tolerogenic artificial antigen-presenting cell (aAPC)s for the induction of $\mathrm{CD}^{+}$and $\mathrm{CD}^{+}{ }^{+} \mathrm{Foxp}^{+}$Tregs in vivo. aAPC NPs were also engineered to promote the tolerogenic activity of NK cells. Those acellular aAPCs were then tested for therapeutic efficacy in vivo in lupus-prone BDF1 mice.

Results The aAPC NPs induced $\mathrm{CD}^{+}$and $\mathrm{CD} 8^{+}$Tregs in vivo in BDF1 mice and protected the animals from lupus disease manifestations. At the NK cell level, protection from lupus-like disease could be ascribed to the production of TGF$\beta$ from the NP-induced NK cells. Finally, the use of aAPC NPs in humanized NSG mice expanded Tregs in numbers sufficient to protect mice from a rapidly evolving human antimouse graft-versus-host disease.

Conclusions These studies provide a proof-of-concept for the potential use of PLGA NPs as acellular aAPCs for the induction of therapeutically effective numbers of Tregs in conditions of pathologic deficit such as in SLE.

\section{0 - Genetics}

\section{GENETICS OF AGE AT SYSTEMIC LUPUS ERYTHEMATOSUS DIAGNOSIS}

${ }^{1}$ Raffaella Carlomagno, ${ }^{1}$ Fangming Liao, ${ }^{2}$ Jingjing Cao, 'Daniela Dominguez, ${ }^{3}$ Dafna D Gladman, ${ }^{4}$ Mariko Ishimori, ${ }^{4}$ Caroline Jefferies, ${ }^{5}$ Diane L Kamen, ${ }^{6}$ Sylvia Kamphuis, ${ }^{7}$ Marisa S Klein-Gitelman, ${ }^{1}$ Andrea M Knight, ${ }^{4}$ Chia-Chi I Lee, ${ }^{1}$ Deborah M Levy, ${ }^{8}$ Karen B Onel, ${ }^{2}$ Andrew D Paterson, ${ }^{9}$ Christine A Peschken, ${ }^{10}$ Janet E Pope, ${ }^{3}$ Zahi Touma, ${ }^{3}$ Murray B Urowitz, ${ }^{4}$ Daniel J Wallace, ${ }^{1}$ Declan Webber, ${ }^{3} J o a n$ E Wither, ${ }^{1,}{ }^{11}$ Earl D Silverman, ${ }^{1}$ ${ }^{2}$ Linda T Hiraki*. 'Division of Rheumatology; ${ }^{2}$ Genetics and Genome Biology, Research Institute, The Hospital for Sick Children; ${ }^{3}$ Schroeder Arthritis Institute, Krembil Research Institute, Toronto Western Hospital, Toronto, Canada; ${ }^{4}$ Division of Rheumatology, Department of Medicine, Cedars Sinai Medical Center, Los Angeles; ${ }^{5}$ Division of Rheumatology and Immunology, Medical University of South Carolina, Charleston, USA; ${ }^{6}$ Department of Pediatric Rheumatology, Sophia Children's Hospital, Erasmus University Medical Center, Rotterdam, Netherlands; ${ }^{7}$ Division of Rheumatology, Department of Pediatrics, Ann and Robert H. Lurie Children's Hospital of Chicago, Chicago; ${ }^{8}$ Pediatric Rheumatology, Hospital for Special Surgery, New York, USA; ${ }^{9}$ Departments of Medicine and Community Health Sciences, University of Manitoba, Winnipeg; ${ }^{10}$ Department of Medicine, University of Western Ontario, St. Joseph's Health Centre, London; ${ }^{11}$ Division of Translational Medicine Research Institute, The Hospital for Sick Children, Toronto, Canada

\subsection{6/lupus-2021-lupus21century.84}

Background Genome wide association studies (GWAS) have identified >100 SNPs associated with systemic lupus erythematosus (SLE) risk. There may be additional loci impacting the age of diagnosis. The purpose of this study was to identify genetic variants associated with age of SLE diagnosis.

Methods Our cohort included patients from tertiary care centres who met ACR and/or SLICC SLE classification criteria. We censored patients missing age at diagnosis. Patients were genotyped on the Illumina Multiethnic Array (MEGA) or Global Screen Array (GSA). Ungenotyped SNPs were imputed using the TopMed reference. We restricted to SNPs with a minor allele frequency (MAF) $\geq 0.01$ and imputation quality $\mathrm{R}^{2} \geq 0.3$. Ancestry was genetically inferred using principal components (PCs) and ADMIXTURE in reference to 1000 Genome Project. We completed genome-wide linear regression of log-transformed age at SLE diagnosis with GENESIS (significance $\left.\mathrm{P}<5 \times 10^{-8}\right)$. Multivariate models adjusted for sex and 5 PCs. We also conducted a GWAS of childhood-onset SLE

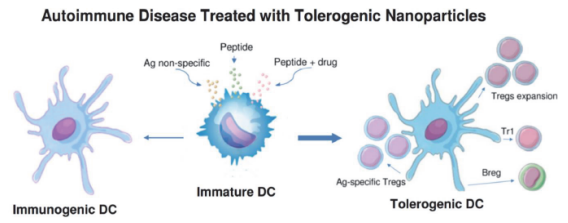


(cSLE) patients, defined as diagnosis $<18$ years of age, vs. adult-onset SLE (aSLE), using logistic regression, and adjusted for the same covariates. We conducted sensitivity analyses where we stratified GWAS by cSLE and aSLE, then meta-analyzed results using inverse variance weighting, as well as ancestry-stratified analyses (Europeans, East Asians, Africans, Amerindians and Admixed).

Results Our cohort included 1489 patients, 761 (51\%) cSLE, $1306(88 \%)$ female. Median age at diagnosis was 17.7 years (IQR 14, 31) in the total cohort, 14.1 years (IQR 11.8, 15.8) in cSLE, and 31.2 years (IQR 24.7, 42) in aSLE. In the total cohort, 576 (39\%) were of European ancestry, 278 (19\%) East Asian, and 253 (17\%) Admixed. We included 11.7M SNPs in GWAS. In the age of SLE diagnosis GWAS, 2 loci on chr16 were genome-wide significantly associated with younger age at diagnosis (top SNP rs11641349, Beta $-0.03 \mathrm{y}$, SE $0.15 \mathrm{y}$, $\mathrm{P}=4.33 \times 10^{-8}$, MAF 0.2). Both SNPs were intronic to CCDC113, a component of centriolar satellites. These loci were also the most significant in the GWAS of cSLE (top SNP rs16959933, OR 1.75 [95\% CI: 1.43, 2.14, P=5.45 x10-8]). Sensitivity analyses showed similar results, yet they did not reach genome-wide significance with top SNPs rs11641349 $\left(\mathrm{P}=3.84 \times 10^{-7}\right)$ and $\operatorname{rs} 16959933\left(\mathrm{P}=4.50 \times 10^{-7}\right)$ in the age group model, and rs11641349 $\left(\mathrm{P}=3.52 \times 10^{-7}\right)$ and $\mathrm{rs} 16959933$ $\left(\mathrm{P}=4.22 \times 10^{-7}\right)$ in the ancestry model.

Conclusion In our multiethnic cSLE and aSLE cohort, we identified genome-wide significant loci associated with age at diagnosis and cSLE risk, intronic to CCDC113. Our study requires independent validation.

\section{GENETIC PREDISPOSITION TO LUPUS ACROSS ANCESTRIES HAS $>300$ SEPARABLE GENETIC CONTRIBUTIONS: WHAT WE KNOW TODAY}

${ }^{1}$ Viktoryia Laurynenka, 1,2Leah C Kottyan, 1,2 Matthew T Weirauch, 1,2,3 Kenneth M Kaufman, ${ }^{3} J o h n$ B Harley*. 'Center for Autoimmune Genomics and Etiology, Cincinnati Children's Hospital Medical Center, Cincinnati, Ohio, USA; ${ }^{2}$ University of Cincinnati College of Medicine, Cincinnati, Ohio, USA; ${ }^{3}$ Research Service, US Department of Veterans Affairs (USDVA) Medical Center, Cincinnati, Ohio, USA

\subsection{6/lupus-2021-lupus21century.85}

Background Systemic lupus erythematosus (SLE) is a complex disease caused by interplay of genetic predisposition and environmental triggers. Strong genetic component in lupus supported by familial clustering, where $10-12 \%$ of SLE patients have an affected first-degree relative, and high concordance rates (24-69\%) in monozygotic twins compared to dizygotic twins and non-twin siblings (2-9\%). In the majority of SLE cases, excluding rare monogenic forms, the genetic components are composed of many polymorphisms with small effects, acting additively, which explain about $44 \%$ to $66 \%$ of the disease etiology. The prevalence and severity of the disease differ across populations, where Africans, Hispanics and East Asians having three to four times higher incidence compared to Europeans, which may be partially explained by genetic differences, as we show here. We summarized all the genetic findings in candidate gene and genome wide association studies (GWAS) for non-monogenic forms of lupus across different populations now available in the medical literature.

Methods A literature search for GWAS and candidate gene studies in SLE was done in the electronic article database PubMed (www.ncbi.nlm.nih.gov/pubmed), NHGRI-EBI GWAS catalog and the references of selected original publications and review articles. We included 127 studies that reported SLE-associated polymorphisms reaching the threshold of significance $\leq 5 \times 10^{-8}$. Variants in linkage disequilibrium (LD) with $\mathrm{r}^{2} \geq 0.8$ were grouped into the same loci. The leading variant of the locus was defined as a variant with lowest $\mathrm{p}$ value. The criteria for the independence of loci were $\mathrm{r}^{2}<0.2$ between leading variants or literature support for there being two (or more) distinguishable contributions to genetic risk.

Results In total we found 730 polymorphisms with $\mathrm{p}$ value between $5 \times 10^{-8}$ and $2.2 \times 10^{-298}$ associated with SLE in Europeans (EU), Asians (AS), African-American (AA) and Mixed Americans/Hispanics (MA). Reported odds ratio (OR) varied between 1.1 and 5, but the majority of the associations are weak effects $(\sim 77 \%$ have $\mathrm{OR}<1.5$ while $\sim 7.7 \%$ have an $\mathrm{OR} \geq 2$ ). These variants we grouped into 315 independent loci: 106 loci in EU, 216 loci in AS, 11 loci in AA, 18 loci in MA and 28 loci was reported only in multiancestral group. Many loci are ancestry specific: 174 (80.56\%) loci are found to date only in AS, 60 (56.60\%) loci only in EU, 3 (27.27\%) loci only in AA, and $3(16.67 \%)$ loci only in MA (figure 1). This finding may be explained by the different allele frequencies in the populations studied. This population specificity of the disease loci could influence on SLE prevalence and be a source for heterogeneity of symptoms and disease severity across populations. Identifying the true causal variants and predicting their function is not a trivial task, as genetic variants are in linkage blocks; therefore, the variant with lowest $\mathrm{p}$ value may not be causal. Among 730 SLE associated polymorphisms only $21(4.52 \%)$ lead to amino acid change, 484 $(66.3 \%)$ lay within gene coding region of 272 genes and the rest are intergenic, which suggests that the majority of SLE associations affect gene regulation, instead of protein sequence. These variants may regulate gene or genes near or far, making the identification of their targets that are involved in mechanisms that change lupus risk a major challenge. The pathway analysis for the 272 genes intersected by SLE variants shows that they are involved in immune processes including responses to pathogens and transcription (MHC class II receptor activity, toll-like receptor signaling, response to cytokines and regulation of their production, immune cell activation and proliferation, DNA binding, transcription regulation, EpsteinBarr virus infection and many others).

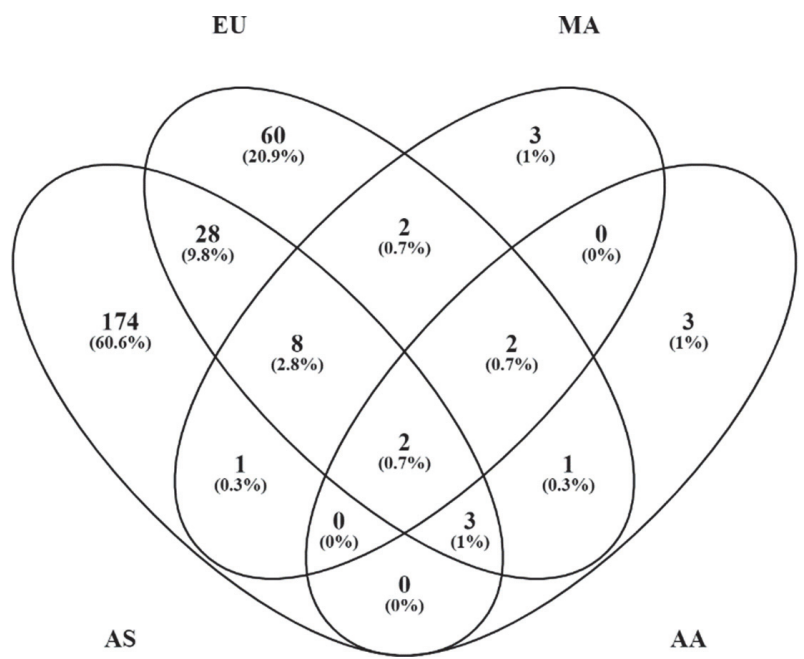

Abstract 1502 Figure 1 